PROCEEDINGS OF THE

AMERICAN MATHEMATICAL SOCIETY

Volume 138, Number 11, November 2010, Pages 4089-4102

S 0002-9939(2010)10431-8

Article electronically published on June 16, 2010

\title{
SURFACES EXPANDING BY THE POWER OF THE GAUSS CURVATURE FLOW
}

\author{
QI-RUI LI
}

(Communicated by Richard A. Wentworth)

\begin{abstract}
In this paper, we describe the flow of 2-surfaces in $\mathbb{R}^{3}$ for some negative power of the Gauss curvature. We show that strictly convex surfaces expanding with normal velocity $K^{-\alpha}$, when $\frac{1}{2}<\alpha \leq 1$, converge to infinity in finite time. After appropriate rescaling, they converge to spheres. In the 2-dimensional case, our results close an apparent gap in the powers considered by previous authors, that is, for $\alpha \in\left(0, \frac{1}{2}\right]$ by Urbas and Huisken and for $\alpha=1$ by Schnürer.
\end{abstract}

\section{INTRODUCTION}

Let $X(\cdot, 0): M \rightarrow \mathbb{R}^{3}$ be a closed strictly convex surface with $M_{0}=X(\cdot, 0)(M)$. Considering $X(\cdot, t): M \rightarrow \mathbb{R}^{3}$, we suppose that $M_{t}=X(\cdot, t)(M) \subset \mathbb{R}^{3}$ satisfy

$$
\frac{\partial}{\partial t} X=\frac{1}{K^{\alpha}} \nu,
$$

where we choose $\nu$ to be the outer unit normal vector of $M_{t}$. For $0<\alpha<+\infty$, it is known by [10] that this is a parabolic flow equation. Hence it has a solution on a maximal time interval. It is also known that $M_{t}$ remain strictly convex during the flow if the initial data is strictly convex.

The flow $\dot{X}=K^{-\alpha} \nu$ with positive $\alpha$ has been taken into account by many authors. It was first studied by Huisken [11] and Urbas [16] in the case $\alpha \in\left(0, \frac{1}{n}\right]$ ( $n$ is the dimension of the hypersurface and $n \geq 2$ ). The basic result is that for $\alpha$ in this range any smooth compact strictly convex initial hypersurface evolves under the flow and expands to infinity as $t \rightarrow+\infty$. The hypersurfaces $M_{t}$ remain smooth and strictly convex and after appropriate rescaling they converge to a sphere with centre at the origin. For the case $\alpha=1$, Schnürer [13] considered the surfaces expanding by the inverse Gauss curvature flow and obtained a similar result. He also considered other velocities, for example, $\frac{H^{\alpha}}{K^{\alpha}}, \alpha=1,2,3$, and $\frac{|A|^{2}}{K^{2}}$, and obtained nice results. On the other hand, Urbas 15 has classified all complete noncompact embedded convex hypersurfaces in $\mathbb{R}^{n+1}$ which move homothetically under flow by some negative power of Gauss curvature.

For the contracting case, there are also some interesting aspects of flow by positive powers of Gauss curvature. The flow $\dot{X}=-K^{\alpha} \nu$ with $\alpha=1$ was originally

Received by the editors June 2, 2009 and, in revised form, October 9, 2009; November 26, 2009; December 19, 2009; December 31, 2009 and February 6, 2010.

2010 Mathematics Subject Classification. Primary 53C44, 35K55.

Key words and phrases. Surface, expanding curvature flow, velocity function $K^{-\alpha}$.

(c)2010 American Mathematical Society Reverts to public domain 28 years from publication 4089 
proposed by Firey [6] in 1974 as a model for the wearing of a convex body, such as a stone worn by abrasive forces. The first significant progress on this problem was made in 1985 by Tso [14, who showed that if $M_{0}$ is smooth and strictly convex, then the flow has a smooth solution up to a finite time $T$, such that the hypersurfaces $M_{t}$ converge to a point as $t \rightarrow T$. Later Chow [3] studied the case $\alpha=\frac{1}{n}(n$ is the dimension of the hypersurface) and obtained a similar result as Tso and, in addition, showed that the asymptotic shape was indeed a sphere. He also proved some partial results for other values of $\alpha$. Chow's result is analogous to the wellknown result of Huisken [9] for the mean curvature flow. And then Andrews [2] obtained analogous results for compact convex hypersurfaces moving by a general curvature function having the same homogeneity as the mean curvature. Andrews 1] finally solved Firey's 1974 conjecture that convex surfaces moving by their Gauss curvature become spherical as they contract to points.

It has been mentioned above that Urbas [16] showed that if $0<\alpha \leq \frac{1}{n}$, any smooth compact strictly convex initial $n$-dimensional hypersurface evolves under the flow and expands to infinity as $t \rightarrow+\infty$. After a suitable rescaling they converge to a sphere. But for $\alpha \geq \frac{1}{n}$, the problem is still open. Our paper concerns a 2-dimensional surface for the case $\frac{1}{2}<\alpha \leq 1$. It is the complement for Urbas [16] and Schnürer [13. The method we used in this paper is derived from [13]. For $0<\alpha \leq \frac{1}{2}$, we give another proof of the result in [16].

Our main theorem is

Theorem 1.1. When $\frac{1}{2}<\alpha \leq 1$, for any smooth closed strictly convex surface $M$ in $\mathbb{R}^{3}$, there exists a smooth family of surfaces $M_{t}, t \in[0, T), 0<T<+\infty$, solving equation (1.1) with $M_{0}=M$. For $t \rightarrow T$, the surfaces $M_{t}$ converge to infinity. Furthermore, the rescaled surfaces $\tilde{M}_{t}=\left\{(2 \alpha-1) T-(2 \alpha-1) t^{\frac{1}{2 \alpha-1}} M_{t}\right.$ converge smoothly to the unit sphere $\mathbb{S}^{2}$.

To get our result, the key step is to show the ratio of two principal curvatures $\lambda_{1}$ and $\lambda_{2}$ satisfies a pinching condition, that is, $0<\frac{1}{\beta}<\frac{\lambda_{1}}{\lambda_{2}}<\beta$ for some positive constant $\beta$. We find a simple but powerful monotone quantity,

$$
\max _{M_{t}} \frac{\left(2|A|^{2}-H^{2}\right)}{\left(H^{2}-|A|^{2}\right)^{1+\alpha}},
$$

non-increasing in time, from which we achieve our ratio pinching estimate as desired.

The following examples show the motivation of establishing our theorem.

Example 1.2. When $\alpha=\frac{1}{2}$, spheres $\partial B_{\rho(t)}\left(x_{0}\right)$ solve (1.1) for $t \in[0,+\infty)$ with $\rho(t)=\rho_{0} e^{t}$ and $\rho_{0}$ is the initial radius.

Example 1.3. When $0<\alpha<\frac{1}{2}$, spheres $\partial B_{\rho(t)}\left(x_{0}\right)$ solve (1.1) for $t \in[0,+\infty)$ with $\rho(t)=\left\{(1-2 \alpha) t+\rho_{0}^{1-2 \alpha}\right\}^{\frac{1}{1-2 \alpha}}$ and $\rho_{0}$ is the initial radius.

Example 1.4. When $\alpha>\frac{1}{2}$, spheres $\partial B_{\rho(t)}\left(x_{0}\right)$ solve (1.1) for $t \in[0, T)$ with $\rho(t)=\{(2 \alpha-1) T-(2 \alpha-1) t\}^{-\frac{1}{2 \alpha-1}}$, where $T=\frac{\rho_{0}^{1-2 \alpha}}{2 \alpha-1}$ and $\rho_{0}$ is the initial radius.

The rest of this paper is organized as follows. In Section 2, we explain our notation. Section 3 concerns the key step, the proof of the monotonicity of our test function (1.2) during the flow. We prove convergence to infinity for appropriate points on the surfaces in Section 4, and we also obtain that the surfaces converge to 
infinity and, after appropriate rescaling, to a round sphere in this section. Section 5 concerns the smooth convergence. This finishes the proof of our main theorem. The case $0<\alpha \leq 1 / 2$ is contained in Section 6 , where we provide a different proof from Urbas in the 2-dimensional case.

\section{Notation}

We use $\left(g_{i j}\right)$ and $\left(h_{i j}\right)$ to denote the induced metric and the second fundamental form respectively. Here Einstein summation convention is adopted. Indices are raised and lowered with respect to the metric or its inverse $\left(g^{i j}\right)$. The inverse of the second fundamental form is denoted by $\left(\tilde{h}^{i j}\right)$. The principal curvatures $\lambda_{1}, \lambda_{2}$ are the eigenvalues of the second fundamental form with respect to the metric. A surface is called strictly convex if all principal curvatures are positive. We will assume this throughout the paper.

Some standard notations are used: they are the mean curvature $H=\lambda_{1}+\lambda_{2}$, the square of the norm of the second fundamental form $|A|^{2}=\lambda_{1}^{2}+\lambda_{2}^{2}, \operatorname{tr} A^{k}=\lambda_{1}^{k}+\lambda_{2}^{k}$, and the Gauss curvature $K=\lambda_{1} \lambda_{2}$. We write indices, preceded by semicolons, to indicate covariant differentiation with respect to the induced metric. It's wellknown that one can choose coordinate systems such that, at a fixed point, the metric tensor equals the Kronecker delta, $g_{i j}=\delta_{i j}$, and the second fundamental form $\left(h_{i j}\right)=\operatorname{diag}\left(\lambda_{1}, \lambda_{2}\right)$ for convenience. We'll make use of this kind of coordinate system repeatedly during the computation.

Let's consider the general case. Let $X_{t}=X(x, t): M^{2} \rightarrow \mathbb{R}^{3}$ be a family of surfaces moving according to $\frac{\partial}{\partial t} X=-F \nu$. The normal velocity $F$ can be considered as a function of $\left(\lambda_{1}, \lambda_{2}\right)$. We set $F^{i j}=\frac{\partial F}{\partial h_{i j}}, F^{i j, k l}=\frac{\partial^{2} F}{\partial h_{i j} \partial h_{k l}}$. Note that in a coordinate system with diagonal $h_{i j}$ and $g_{i j}=\delta_{i j}$ as mentioned above, $F^{i j}$ is diagonal. Especially when $F=-K^{-\alpha}$ has been set, one can obtain $F^{i j}=\alpha K^{-\alpha} \tilde{h}^{i j}$ directly.

Recall (see e.g. [9, 10]) that for a hypersurface moving according to $\frac{\partial}{\partial t} X=-F \nu$, we have the following results:

$$
\begin{gathered}
\frac{\partial}{\partial t} g_{i j}=-2 F h_{i j} \\
\frac{\partial}{\partial t} h_{i j}=F_{; i j}-F h_{i}^{k} h_{k j}, \\
\frac{\partial}{\partial t} \nu^{\alpha}=g^{i j} F_{; i} X_{; j}^{\alpha},
\end{gathered}
$$

where we use Greek indices to refer to components in the ambient space $\mathbb{R}^{3}$. For the sake of computing evolution equations, we will need the Gauss equation and the Ricci identity for the second fundamental form:

$$
\begin{aligned}
R_{i j k l} & =h_{i k} h_{j l}-h_{i l} h_{j k}, \\
h_{i k ; l j} & =h_{i k ; j l}+h_{k}^{a} R_{a i l j}+h_{i}^{a} R_{a k l j} .
\end{aligned}
$$

The Gauss formula and the Weingarten equation

$$
X_{; i j}^{\alpha}=-h_{i j} \nu^{\alpha} \quad \text { and } \nu_{; i}^{\alpha}=h_{i}^{k} X_{; k}^{\alpha}
$$

will be employed in our computation.

Finally, we introduce $c$ to denote universal, estimated constants. 


\section{Monotone QUANTITY}

We want to show that (1.2) is a monotone quantity; in fact it is non-increasing in time.

We begin with some illustration and computation. Suppose the testing function $w$ is symmetric of two principal curvatures. In the case here, we can represent each curvature as some function of mean curvature $H$ and the square of the norm of the second fundamental form $|A|^{2}$. We can do the same with $w$. Once we need to achieve the monotonicity of $w$, we just evaluate the evolution equation of test quantity $w$ at a critical point and apply the maximum principle.

More precisely, according to [13, we obtain the following calculations at a critical point of $w$ :

$$
\frac{\partial}{\partial t} w-F^{i j} w_{; i j}=C_{w}\left(\lambda_{1}, \lambda_{2}\right)+G_{w}\left(\lambda_{1}, \lambda_{2}\right) h_{11 ; 1}^{2}+G_{w}\left(\lambda_{2}, \lambda_{1}\right) h_{22 ; 2}^{2},
$$

where

$$
\begin{aligned}
C_{w}= & \frac{\partial w}{\partial H} C_{H}+\frac{\partial w}{\partial|A|^{2}} C_{|A|^{2}}, \\
G_{w}= & \frac{\partial w}{\partial H} G_{H}+\frac{\partial w}{\partial|A|^{2}} G_{|A|^{2}}-\frac{\partial^{2} w}{\partial H \partial H} \frac{\partial F}{\partial \lambda_{1}}(1+\kappa)^{2} \\
& -4 \frac{\partial^{2} w}{\partial|A|^{2} \partial|A|^{2}} \frac{\partial F}{\partial \lambda_{1}}\left(\lambda_{1}+\kappa \lambda_{2}\right)^{2} \\
& -4 \frac{\partial^{2} w}{\partial H \partial|A|^{2}} \frac{\partial F}{\partial \lambda_{1}}(1+\kappa)\left(\lambda_{1}+\kappa \lambda_{2}\right),
\end{aligned}
$$

and $\kappa, C_{H}, C_{|A|^{2}}, G_{H}, G_{|A|^{2}}$ are determined by

$$
\begin{aligned}
\kappa= & -\frac{\partial w}{\partial \lambda_{1}} / \frac{\partial w}{\partial \lambda_{2}}, \\
C_{H}= & \left(\frac{\partial F}{\partial \lambda_{1}} \lambda_{1}^{2}+\frac{\partial F}{\partial \lambda_{2}} \lambda_{2}^{2}\right) H+\left(F-\frac{\partial F}{\partial \lambda_{1}} \lambda_{1}-\frac{\partial F}{\partial \lambda_{2}} \lambda_{2}\right)|A|^{2}, \\
G_{H}= & \frac{\partial^{2} F}{\partial \lambda_{1} \partial \lambda_{1}}+2 \frac{\partial^{2} F}{\partial \lambda_{1} \partial \lambda_{2}} \kappa+\frac{\partial^{2} F}{\partial \lambda_{2} \partial \lambda_{2}} \kappa^{2}+2 \frac{\frac{\partial F}{\partial \lambda_{1}}-\frac{\partial F}{\partial \lambda_{2}}}{\lambda_{1}-\lambda_{2}} \kappa^{2}, \\
C_{|A|^{2}}= & 2\left(\frac{\partial F}{\partial \lambda_{1}} \lambda_{1}^{2}+\frac{\partial F}{\partial \lambda_{2}} \lambda_{2}^{2}\right)|A|^{2}+2\left(F-\frac{\partial F}{\partial \lambda_{1}} \lambda_{1}-\frac{\partial F}{\partial \lambda_{2}} \lambda_{2}\right) t r A^{3}, \\
G_{|A|^{2}}= & -2\left(\frac{\partial F}{\partial \lambda_{1}}\left(1+\kappa^{2}\right)+2 \frac{\partial F}{\partial \lambda_{2}} \kappa^{2}\right) \\
& +2\left(\frac{\partial^{2} F}{\partial \lambda_{1} \partial \lambda_{1}}+2 \frac{\partial^{2} F}{\partial \lambda_{1} \partial \lambda_{2}} \kappa+\frac{\partial^{2} F}{\partial \lambda_{2} \partial \lambda_{2}} \kappa^{2}\right) \lambda_{1}+4 \frac{\frac{\partial F}{\partial \lambda_{1}}-\frac{\partial F}{\partial \lambda_{2}}}{\lambda_{1}-\lambda_{2}} \kappa^{2} \lambda_{2} .
\end{aligned}
$$

During the calculation, (2.1), (2.2), (2.4), (2.5) are employed, and (see [2, 7]) one has

$$
F^{i j, k l} \eta_{i j} \eta_{k l}=\sum \frac{\partial^{2} F}{\partial \lambda_{i} \partial \lambda_{j}} \eta_{i i} \eta_{j j}+\sum_{i \neq j} \frac{\frac{\partial F}{\partial \lambda_{i}}-\frac{\partial F}{\partial \lambda_{j}}}{\lambda_{i}-\lambda_{j}} \eta_{i j}^{2},
$$

for symmetric matrices $\left(\eta_{i j}\right)$ and $\lambda_{1} \neq \lambda_{2}$; or if $\lambda_{1}=\lambda_{2}$, then the last term is interpreted as a limit.

The calculation above, though tedious, is straightforward. In [13, the author constructs a monotone quantity and eventually deduces the pinching estimate for 
principal curvatures from his monotone quantity. Unfortunately, the quantity there seems to be invalid in our case. However, based on some modifications, we construct another quantity. In our case, the computation becomes much longer since the test function $w$ itself is complicated. Fortunately, the formula above allows us to write a program. It is possible for the author to efficiently and expediently evaluate the evolution equation of various test functions under all kinds of curvature flow through computer coding.

Now, we state our theorem.

Theorem 3.1. For a family of smooth closed strictly convex surfaces $M_{t}$ in $\mathbb{R}^{3}$ flowing according to $\dot{X}=K^{-\alpha} \nu$, where $0<\alpha \leq 1$,

$$
\max _{M_{t}} \frac{\left(\lambda_{1}-\lambda_{2}\right)^{2}}{2^{1+\alpha} \lambda_{1}^{1+\alpha} \lambda_{2}^{1+\alpha}}=\max _{M_{t}} w=\max _{M_{t}} \frac{\left(2|A|^{2}-H^{2}\right)}{\left(H^{2}-|A|^{2}\right)^{1+\alpha}}
$$

is non-increasing in time.

Proof. Set $F=-K^{-\alpha}$ and $w=\frac{\left(2|A|^{2}-H^{2}\right)}{\left(H^{2}-|A|^{2}\right)^{1+\alpha}}$. Employing equations (3.1), (3.2), (3.3) and $\frac{\partial F}{\partial \lambda_{k}}=\alpha K^{-\alpha} / \lambda_{k}$, we achieve that

$$
\begin{aligned}
& \frac{\partial w}{\partial t}-F^{i j} w_{i j} \\
& \quad=-\frac{\alpha+1}{2^{\alpha+1} \lambda_{1}^{2 \alpha+1} \lambda_{2}^{2 \alpha+1}}\left(\lambda_{1}+\lambda_{2}\right)\left(\lambda_{1}-\lambda_{2}\right)^{2} \\
& \quad-\frac{h_{11 ; 1}^{2}}{2^{\alpha} \lambda_{1}^{2 \alpha+4} \lambda_{2}^{2 \alpha+1}\left(\alpha \lambda_{1}+\lambda_{1}-\alpha \lambda_{2}+\lambda_{2}\right)^{2}}\left\{\alpha \left(\left(1-\alpha^{2}\right) \lambda_{1}^{4}\right.\right. \\
& \left.\left.\quad+4\left(1-\alpha^{2}\right) \lambda_{1}^{3} \lambda_{2}+\left(6+10 \alpha^{2}\right) \lambda_{1}^{2} \lambda_{2}^{2}+4\left(1-\alpha^{2}\right) \lambda_{1} \lambda_{2}^{3}+\left(1-\alpha^{2}\right) \lambda_{2}^{4}\right)\right\} \\
& \quad-\frac{h_{22 ; 2}^{2}}{2^{\alpha} \lambda_{1}^{2 \alpha+1} \lambda_{2}^{2 \alpha+4}\left(\lambda_{1}-\alpha \lambda_{1}+\alpha \lambda_{2}+\lambda_{2}\right)^{2}}\left\{\alpha \left(\left(1-\alpha^{2}\right) \lambda_{2}^{4}\right.\right. \\
& \left.\left.\quad+4\left(1-\alpha^{2}\right) \lambda_{1} \lambda_{2}^{3}+\left(6+10 \alpha^{2}\right) \lambda_{1}^{2} \lambda_{2}^{2}+4\left(1-\alpha^{2}\right) \lambda_{1}^{3} \lambda_{2}+\left(1-\alpha^{2}\right) \lambda_{1}^{4}\right)\right\} .
\end{aligned}
$$

Since $0<\alpha \leq 1$, we obtain

$$
\frac{\partial w}{\partial t}-F^{i j} w_{; i j} \leq 0
$$

Our theorem follows by applying the maximum principle.

\section{Convergence to sphere}

According to [10], (1.1) is a parabolic evolution equation for strictly convex initial data and it has a solution on a maximal time interval $[0, T), 0<T \leq+\infty$.

In this section, we shall first show that some points on $M_{t}$ must converge to infinity for $t \rightarrow T<+\infty$, i.e.

$$
\limsup _{t \rightarrow T} \sup _{M_{t}}|X|=+\infty
$$

Then we show that $M_{t}$ converge to a sphere after appropriate rescaling by virtue of the Aleksandrov reflection principle of Bennett Chow, Robert Gulliver [5] and James McCoy [12, Theorem 3.1], which is our Theorem 4.8. 
We imitate 13] to obtain this conclusion, but some modifications are necessary. Let's assume $\limsup _{t \rightarrow T} \sup _{M_{t}}|X|<R$ for some positive constant $R$ beforehand and derive a contradiction.

We begin with some simple lemmas.

Lemma 4.1 (containment principle). Let $M_{t}$ and $\tilde{M}_{t}$ be two smooth closed strictly convex solutions to (1.1) on some time interval $\left[0, T^{*}\right)$. If $M_{0}$ encloses $\tilde{M}_{0}$, then $M_{t}$ encloses $\tilde{M}_{t}$ at any time $t \in\left[0, T^{*}\right)$ for which both solutions exist.

Proof. This is a standard consequence of the maximum principle.

Lemma 4.2. Let $\frac{1}{2}<\alpha \leq 1$ and $M_{t}$ be a family of smooth closed strictly convex solutions to (1.1) on a maximal time interval $[0, T)$. Then $T<+\infty$.

Proof. Considering the Example 1.4 in Section 1, spheres solving (1.1) tend to infinity in finite time. So the containment principle implies that $T<+\infty$.

Then we show that the principal curvatures of $M_{t}$ stay uniformly bounded above under our curvature flow.

Lemma 4.3. For a smooth closed strictly convex surface $M$ in $\mathbb{R}^{3}$, flowing according to $\dot{X}=K^{-\alpha} \nu$, where $\alpha \in(0,+\infty)$, there exists a positive constant $\mu$ such that two principal curvatures satisfy $\max \left\{\lambda_{1}, \lambda_{2}\right\} \leq \mu$ during the flow.

Proof. Consider $M_{i j}:=h_{i j}-\mu g_{i j}$, where $\mu>0$ is chosen large enough such that $M_{i j}$ is negative semi-definite initially. It is sufficient to show that $M_{i j}$ remains negative semi-definite.

Employing equations (2.1), (2.2), (2.4) and (2.5), we obtain the following evolution equations for a family $M_{t}$ of surfaces flowing according to $\dot{X}=-F \nu$ :

$$
\begin{aligned}
& \frac{\partial}{\partial t} M_{i j}-F^{k l} M_{i j ; k l} \\
& \quad=F^{k l} h_{k}^{a} h_{a l} \cdot h_{i j}-F^{k l} h_{k l} \cdot h_{i}^{a} h_{a j}-F h_{i}^{k} h_{k j}+2 \mu F h_{i j} \\
& \quad+F^{k l, r s} h_{k l ; i} h_{r s ; j} .
\end{aligned}
$$

Set $F=-K^{-\alpha}$. Note that in this case, $F^{k l, r s} h_{k l ; i} h_{r s ; j}$ is negative semi-definite. Hence we have

$$
\frac{\partial}{\partial t} M_{i j}-F^{k l} M_{i j ; k l} \leq F^{k l} h_{k}^{a} h_{a l} \cdot h_{i j}-F^{k l} h_{k l} \cdot h_{i}^{a} h_{a j}-F h_{i}^{k} h_{k j}+2 \mu F h_{i j} .
$$

Let $\xi$ be a zero eigenvalue of $M_{i j}$ with $|\xi|=1, M_{i j} \xi^{j}=h_{i j} \xi^{j}-\mu g_{i j} \xi^{j}$, i.e. $h_{i j} \xi^{i} \xi^{j}=$ $\mu g_{i j} \xi^{i} \xi^{j}=\mu$. So we obtain, at the point with $M_{i j} \leq 0$,

$$
\begin{aligned}
& \left(F^{k l} h_{k}^{a} h_{a l} \cdot h_{i j}-F^{k l} h_{k l} \cdot h_{i}^{a} h_{a j}-F h_{i}^{k} h_{k j}+2 \mu F h_{i j}\right) \xi^{i} \xi^{j} \\
& \quad=(H-2 \mu) \alpha \mu K^{-\alpha}-\mu^{2} K^{-\alpha} \\
& \quad \leq-\mu^{2} K^{-\alpha} \\
& \quad \leq 0
\end{aligned}
$$

and the maximum principle for tensors $[4,8$, implies the claimed result.

We now deduce our pinching estimate. 
Lemma 4.4. For a smooth closed strictly convex surface $M_{t}$ in $\mathbb{R}^{3}$, flowing according to $\dot{X}=K^{-\alpha} \nu$, where $0<\alpha \leq 1$, there exists $\beta=\beta\left(M_{0}\right)$ such that $0<\frac{1}{\beta} \leq \frac{\lambda_{1}}{\lambda_{2}} \leq \beta$.

Proof. Theorem 3.1 and Lemma 4.3 imply that

$$
\frac{\left(\frac{\lambda_{1}}{\lambda_{2}}-1\right)^{2}}{2^{1+\alpha} \mu^{2 \alpha} \frac{\lambda_{1}}{\lambda_{2}}}=\frac{\left(\lambda_{1}-\lambda_{2}\right)^{2}}{2^{1+\alpha} \mu^{2 \alpha} \lambda_{1} \lambda_{2}} \leq \frac{\left(\lambda_{1}-\lambda_{2}\right)^{2}}{2^{1+\alpha} \lambda_{1}^{1+\alpha} \lambda_{2}^{1+\alpha}}<c .
$$

Thus we obtain the bound on $\frac{\lambda_{1}}{\lambda_{2}}$ claimed above.

The following result shows that $K$ stays uniformly bounded below by a positive constant as long as $M_{t}$ is enclosed by a ball of fixed positive radius. This is the key step which we will use to derive a contradiction if one assumes $\lim \sup |X|<R$ for some positive constant $R$ beforehand.

Lemma 4.5. When $0<\alpha \leq 1$, for a strictly convex solution of (1.1), $K$ is uniformly bounded below by a positive constant in terms of the radius $R$ of an enclosing sphere $\partial B_{R}\left(x_{0}\right)$, the pinching ratio $\lambda_{1} / \lambda_{2}$, and $\max _{M_{0}} \frac{K^{-\alpha}}{2 R^{2}-\left|X-x_{0}\right|^{2}}$. More precisely, we have everywhere

$$
K \geq\left\{\frac{1}{2 R^{2}} \min \left\{\left(\max _{M_{0}} \frac{K^{-\alpha}}{2 R^{2}-\left|X-x_{0}\right|^{2}}\right)^{-1}, \varepsilon^{2 \alpha} R^{2-2 \alpha}\right\}\right\}^{\frac{1}{\alpha}}
$$

where $\varepsilon=\frac{-(2 \alpha+1)+\sqrt{(2 \alpha+1)^{2}+8 \alpha^{2}(1+1 / \beta)}}{2 \alpha(1+\beta)}$ with $\beta$ as in Lemma 4.4 .

Proof. Without loss of generality, we may assume that $x_{0}=0$.

We obtain the following evolution equations for a family $M_{t}$ of surfaces flowing according to $\dot{X}=-F \nu$ by some standard computations:

$$
\begin{gathered}
\frac{\partial}{\partial t} F-F^{i j} F_{; i j}=F F^{i j} h_{i}^{k} h_{k j}, \\
\frac{\partial}{\partial t}|X|^{2}-F^{i j}\left(|X|^{2}\right)_{; i j}=2\left(F^{i j} h_{i j}-F\right)\langle X, \nu\rangle-2 F^{i j} g_{i j} .
\end{gathered}
$$

In a critical point of $\left.\frac{-F}{2 R^{2}-|X|^{2}}\right|_{M_{t}}$, i.e. $\left(2 R^{2}-|X|^{2}\right) F_{; k}+F\left(|X|^{2}\right)_{; k}=0$, employing equations (4.2), (4.3), we obtain

$$
\begin{aligned}
\frac{\partial}{\partial t} & \log \frac{-F}{2 R^{2}-|X|^{2}}-F^{i j}\left(\log \frac{-F}{2 R^{2}-|X|^{2}}\right)_{; i j} \\
& =\frac{1}{F}\left(\frac{\partial}{\partial t} F-F^{i j} F_{; i j}\right)+\frac{1}{2 R^{2}-|X|^{2}}\left(\frac{\partial}{\partial t}|X|^{2}-F^{i j}\left(|X|^{2}\right)_{; i j}\right) \\
& =\frac{1}{2 R^{2}-|X|^{2}}\left\{\left(2 R^{2}-|X|^{2}\right) F^{i j} h_{i}^{k} h_{k j}+2\left(F^{i j} h_{i j}-F\right)\langle X, \nu\rangle-2 F^{i j} g_{i j}\right\} .
\end{aligned}
$$

When $F=-K^{-\alpha}$ has been set, we get

$$
\begin{aligned}
\frac{\partial}{\partial t} & \log \frac{K^{-\alpha}}{2 R^{2}-|X|^{2}}-F^{i j}\left(\log \frac{K^{-\alpha}}{2 R^{2}-|X|^{2}}\right)_{; i j} \\
& =\frac{K^{-\alpha-1}}{2 R^{2}-|X|^{2}}\left\{\alpha\left(2 R^{2}-|X|^{2}\right) H K+2(2 \alpha+1)\langle X, \nu\rangle K-2 \alpha H\right\}
\end{aligned}
$$


Thus we deduce that the following inequality comes into existence in an increasing maximum of $\frac{K^{-\alpha}}{2 R^{2}-|X|^{2}}$ :

$$
\begin{aligned}
0 \leq & \alpha\left(2 R^{2}-|X|^{2}\right) H K+2(2 \alpha+1)\langle X, \nu\rangle K-2 \alpha H \\
& \leq 2 \alpha R^{2} H K+2(2 \alpha+1) R K-2 \alpha H .
\end{aligned}
$$

We rewrite this inequality and apply our pinching estimate $\lambda_{\max } \leq \beta \lambda_{\min }$ as in Lemma 4.4:

$$
\begin{aligned}
4 \alpha \lambda_{\min } & \leq 2 \alpha H \leq 2 \alpha R^{2} H K+2(2 \alpha+1) R K \\
& \leq 2 \alpha R^{2}(1+\beta) \beta \lambda_{\min }^{3}+2(2 \alpha+1) \beta R \lambda_{\min }^{2}
\end{aligned}
$$

thus,

$$
\alpha R^{2}(1+\beta) \beta \lambda_{\min }^{2}+(2 \alpha+1) \beta R \lambda_{\min }-2 \alpha \geq 0
$$

Therefore, we conclude that $\lambda_{\min } \geq \frac{-(2 \alpha+1)+\sqrt{(2 \alpha+1)^{2}+8 \alpha^{2}(1+1 / \beta)}}{2 \alpha(1+\beta) R}=\varepsilon R^{-1}>0$; consequently $\frac{K^{-\alpha}}{2 R^{2}-|X|^{2}} \leq \frac{\varepsilon^{-2 \alpha}}{R^{2-2 \alpha}}$ in an increasing maximum of $\frac{K^{-\alpha}}{2 R^{2}-|X|^{2}}$.

Thus we obtain everywhere

$$
\frac{K^{-\alpha}}{2 R^{2}-|X|^{2}} \leq \max _{M_{t}} \frac{K^{-\alpha}}{2 R^{2}-|X|^{2}} \leq \max \left\{\max _{M_{0}} \frac{K^{-\alpha}}{2 R^{2}-|X|^{2}}, \frac{\varepsilon^{-2 \alpha}}{R^{2-2 \alpha}}\right\},
$$

and then

follows.

$$
K \geq\left\{\frac{1}{2 R^{2}} \min \left\{\left(\max _{M_{0}} \frac{K^{-\alpha}}{2 R^{2}-|X|^{2}}\right)^{-1}, \varepsilon^{2 \alpha} R^{2-2 \alpha}\right\}\right\}^{\frac{1}{\alpha}}
$$

In [13, Lemma 4.3] the author got a lower bound on the Gauss curvature $K$ by considering the quantity $\frac{K^{-1}}{2 R-\left\langle X-x_{0}, \nu\right\rangle}$. As a minor modification, one can consider $\frac{K^{-\alpha}}{2 R-\left\langle X-x_{0}, \nu\right\rangle}$. Unfortunately $\frac{K^{-\alpha}}{2 R-\left\langle X-x_{0}, \nu\right\rangle}$ does not work when $0<\alpha \leq \frac{1}{2}$, which we will use in Section 6.

We now obtain a positive lower bound on the principal curvatures in the case of $\limsup _{t \rightarrow T}|X|<R$ for some positive constant $R$. This fact can be stated as the following lemma.

Lemma 4.6. Under the assumptions of Lemma 4.5, two principal curvatures have positive lower bound.

Proof. The positive lower bound on $K$, Lemma 4.5, and our pinching estimate, Lemma 4.4, imply a positive lower bound on the principal curvatures.

Now, we have already proved our conclusion mentioned at the beginning of this section. Let's express this conclusion as the following theorem. For the details of the proof see [13, Lemma 4.7].

Theorem 4.7. Let $M_{t}$ be a family of smooth closed strictly convex surfaces solving (1.1) on a maximal time interval $[0, T)$ when $\frac{1}{2}<\alpha \leq 1$. Then

$$
\limsup _{t \rightarrow T} \sup _{M_{t}}|X|=+\infty \text {. }
$$

In the discussion above we have showed that some points of the surfaces under our flow converge to infinity. Next we shall show that, in fact, "all" the points converge to infinity in finite time. Furthermore, they converge to a sphere after appropriate rescaling. 
Theorem 4.8. Under the assumptions of Theorem 1.1, we get

$$
\liminf _{t \rightarrow T M_{t}}|X|=+\infty
$$

More precisely, there exists $c=c\left(M_{0}\right)$ such that

$$
\sup _{M_{t}}|X|-c \leq\{(2 \alpha-1) T-(2 \alpha-1) t\}^{-\frac{1}{2 \alpha-1}} \leq \inf _{M_{t}}|X|+c
$$

and

$$
\tilde{M}_{t}=\{(2 \alpha-1) T-(2 \alpha-1) t\}^{\frac{1}{2 \alpha-1}} M_{t} \subset B_{\rho_{+}(t)}(0) \backslash B_{\rho_{-}(t)}(0),
$$

where

$$
\rho_{+}(t)=1+c\{(2 \alpha-1) T-(2 \alpha-1) t\}^{\frac{1}{2 \alpha-1}}
$$

and

$$
\rho_{-}(t)=1-c\{(2 \alpha-1) T-(2 \alpha-1) t\}^{\frac{1}{2 \alpha-1}},
$$

so the rescaled surfaces $\tilde{M}_{t}=\{(2 \alpha-1) T-(2 \alpha-1) t\}^{\frac{1}{2 \alpha-1}} M_{t}$ converge to the unit sphere $\mathbb{S}^{2}$ in Hausdorff distance.

The proof of this theorem is just a slight modification of [13, Lemma 5.1] with respect to the different power $\alpha$. Hence we omit it and refer to the original paper.

\section{Smooth Convergence to SPhere}

Without loss of generality, for any strictly convex solution of (1.1) $X(\cdot, t): M \rightarrow$ $\mathbb{R}^{3}$, we may assume that $\left|X-x_{0}\right| \neq 0$ on $M \times[0, T)$, where $x_{0} \in \mathbb{R}^{3}$ can be regarded as the origin. We then have the following lemma.

Lemma 5.1. When $0<\alpha \leq 1$, for a strictly convex solution of (1.1), there exists a positive constant $\mu$ depending only on the initial data such that

$$
K \leq \frac{\mu}{\left|X-x_{0}\right|^{2}} \text { on } M \times[0, T) .
$$

Proof. We may suppose that $x_{0}=0$. Let $w=|X|^{2}-\frac{\mu}{K}$. Our proof will show that $w$ stays negative during the flow. By virtue of (4.2) and (4.3), the evolution equation of $w$ is given by

$$
\begin{aligned}
& \frac{\partial}{\partial t} w-F^{i j} w_{; i j} \\
& \quad=2(2 \alpha+1) K^{-\alpha}\langle X, \nu\rangle-(2 \alpha+\mu) K^{-\alpha-1} H \\
& \quad+\mu \frac{1-\alpha}{\alpha^{2}} K^{2 \alpha-1} F^{i j} F_{; i} F_{; j}
\end{aligned}
$$

where $F=-K^{-\alpha}$. We may assume that $\mu$ is so large that $w<0$ on $M_{0}$.

Let $t_{0} \in[0, T)$ be the first time that $\max _{M_{t}} w=0$. Choose $\tilde{x} \in M_{t_{0}}$ such that $w\left(\tilde{x}, t_{0}\right)=0$. At this point, using $F^{i j}=\alpha K^{-\alpha} \tilde{h}^{i j}$ and

$$
0=w_{i}=2\left\langle X, X_{; i}\right\rangle+\frac{\mu}{\alpha} K^{-1+\alpha} F_{; i},
$$


we obtain

$$
\begin{aligned}
0 & \leq 2(2 \alpha+1)\langle X, \nu\rangle-(2 \alpha+\mu) \frac{H}{K} \\
& +4 \frac{\alpha(1-\alpha)}{\mu} K \tilde{h}^{i j}\langle X, X ; i\rangle\left\langle X, X_{; j}\right\rangle \\
& \leq 2(2 \alpha+1) \sqrt{\mu} K^{-\frac{1}{2}}-(2 \alpha+\mu) \frac{H}{K} \\
& +4 \alpha(1-\alpha)\left(\frac{1}{\lambda_{1}}+\frac{1}{\lambda_{2}}\right) .
\end{aligned}
$$

Convexity implies that $\sqrt{K} \leq H$. We therefore obtain

$$
0 \leq-\mu+2(2 \alpha+1) \sqrt{\mu}+2 \alpha(1-2 \alpha) .
$$

For $\mu$ sufficiently large, we get a contradiction.

In order to get the smooth convergence result, we show that

$$
\frac{1}{C} \frac{1}{R(t)^{2}} \leq K \leq C \frac{1}{R(t)^{2}} \text { on } M \times[0, T),
$$

where

$$
R(t)=\{(2 \alpha-1) T-(2 \alpha-1) t\}^{-\frac{1}{2 \alpha-1}}
$$

and $\frac{1}{2}<\alpha \leq 1$. We only need to prove (5.1) in a sufficiently small time interval $\left(T_{0}, T\right)$ since it is easy to get the bound of $R(t)^{2} K$ on the compact set $M \times\left[0, T_{0}\right]$.

Notice that $R(t)$ can be sufficiently large when $t$ is close enough to $T$. According to Theorem 4.8, after properly choosing $T_{0} \in[0, T)$, we have

$$
(1-\theta) R(t)<\left|X-x_{0}\right|<(1+\theta) R(t) \text { on } M \times\left[T_{0}, T\right),
$$

for some positive constant $\theta<1$, which depends only on $T_{0}$ and the initial data. Combining Lemma 5.1 with Lemma 4.5 and Theorem 4.8, we derive (5.1) as desired.

Let's rescale our surfaces

$$
\begin{aligned}
\tilde{X}(z, t) & :=\{(2 \alpha-1) T-(2 \alpha-1) t\}^{\frac{1}{2 \alpha-1}} X(z, t) \\
& =\frac{1}{R(t)} X(z, t) .
\end{aligned}
$$

Define a new time function

$$
\tau(t):=-\frac{1}{2 \alpha-1} \log \left(\frac{T-t}{T}\right) .
$$

We use a tilde to denote geometric quantities of the rescaled surfaces. For $\tilde{X}$, we obtain the evolution equation

$$
\frac{\partial}{\partial \tau} \tilde{X}=\tilde{K}^{-\alpha} \nu-\tilde{X}
$$

Note that $\tilde{K}=R^{2} K$. Hence, according to (5.1) we have bounded the speed of (5.2). By the same discussion as in [13, Section 6], we get smooth convergence as desired.

This finishes the proof of Theorem 1.1. 


\section{6. $K^{-\alpha}$-FLOW WHEN $0<\alpha \leq \frac{1}{2}$}

In this section we briefly discuss $K^{-\alpha}$-flow under the circumstance $0<\alpha \leq \frac{1}{2}$. This is just a special case of Urbas [16] with a different method. We want to show, as before, that some points on $M_{t}$ must converge to infinity for $t \rightarrow+\infty$, i.e.

$$
\lim _{t \rightarrow+\infty} \sup _{M_{t}}|X|=+\infty \text {. }
$$

This conclusion is derived by the following discussion.

In the present situation, equation (1.1) is still parabolic. Hence, the containment principle holds. Let $T$ be the maximal time. Then there are two cases.

Case 1. If $T=+\infty$.

Let $\partial B_{\rho(t)}$ be the sphere, mentioned in Section 1, solving (1.1), and at initial time, our surface $M_{0}$ encloses $\partial B_{\rho(0)}$. According to the containment principle, $M_{t}$ encloses $\partial B_{\rho(t)}$ at any time $t \in[0,+\infty)$. Since $\partial B_{\rho(t)}$ converge to infinity in infinite time, so do $M_{t}$. Our conclusion followed in this case.

Case 2. If $0<T<+\infty$.

Similarly, let $\partial B_{\rho(t)}$ be the sphere, mentioned in Section 1, solving (1.1). The difference is, at initial time, our surface $\partial B_{\rho(0)}$ encloses $M_{0}$. If $M_{t}$ converge to infinity when $t \rightarrow T<+\infty$, so do $\partial B_{\rho(t)}$. But this cannot happen according to our examples in Section 1. The contradiction implies

$$
\limsup _{t \rightarrow T}|X|<R<+\infty
$$

for some positive constant $R$ in this case.

We will derive some contradiction to exclude case 2 . Thus our conclusion follows.

Theorem 6.1. Let $M_{t}$ be a family of smooth closed strictly convex surfaces solving (1.1) on a maximal time interval $[0, T), 0<\alpha \leq \frac{1}{2}$. Then $T=+\infty$, and

$$
\lim _{t \rightarrow+\infty} \sup _{M_{t}}|X|=+\infty \text {. }
$$

Proof. We just need to exclude case 2 mentioned at the beginning of this section.

Assume our flow is exactly case 2. Lemma 4.3 and Lemma 4.6 tell us the principal curvatures of $M_{t}$ stay uniformly bounded above and below by positive constants. We derive a contradiction by the same argument as [13, Lemma 4.7].

Next we show the following theorem, which claims our surfaces uniformly converge to infinity in infinite time and, after rescaling, converge to a sphere.

Theorem 6.2. For surfaces $M_{t}, t \in[0,+\infty)$, solving flow equation

$$
\dot{X}=K^{-\alpha} \nu, \text { where } 0<\alpha \leq \frac{1}{2}
$$

with $M_{0}=M$, we have

$$
\lim _{t \rightarrow+\infty} \inf _{t}|X|=+\infty
$$

More precisely,

(i) If $0<\alpha<\frac{1}{2}$, there exist $c=c\left(M_{0}\right)$ and a unique positive constant $\rho$ such that

$$
\sup _{M_{t}}|X|-c \leq\left\{(1-2 \alpha) t+\rho^{1-2 \alpha}\right\}^{\frac{1}{1-2 \alpha}} \leq \inf _{M_{t}}|X|+c
$$


and

$$
\tilde{M}_{t}=\left\{(1-2 \alpha) t+\rho^{1-2 \alpha}\right\}^{-\frac{1}{1-2 \alpha}} M_{t} \subset B_{R_{+}(t)}(0) \backslash B_{R_{-}(t)}(0)
$$

where $R_{+}(t)=1+c\left\{(1-2 \alpha) t+\rho^{1-2 \alpha}\right\}^{-\frac{1}{1-2 \alpha}}$ and $R_{-}(t)=1-c\{(1-2 \alpha) t+$ $\left.\rho^{1-2 \alpha}\right\}^{-\frac{1}{1-2 \alpha}}$, so the rescaled surfaces $\tilde{M}_{t}=\left\{\left[(1-2 \alpha) t+\rho^{1-2 \alpha}\right]^{-\frac{1}{1-2 \alpha}}\right\} M_{t}$ converge to the unit sphere $\mathbb{S}^{2}$.

(ii) If $\alpha=\frac{1}{2}$, there exist $c=c\left(M_{0}\right)$ and a unique positive constant $\rho$ such that

$$
\sup _{M_{t}}|X|-c \leq \rho e^{t} \leq \inf _{M_{t}}|X|+c
$$

and

$$
\tilde{M}_{t}=\rho^{-1} e^{-t} M_{t} \subset B_{1+c \rho^{-1} e^{-t}}(0) \backslash B_{1-c \rho^{-1} e^{-t}}(0),
$$

so the rescaled surfaces $\tilde{M}_{t}=\rho^{-1} e^{-t} M_{t}$ converge to the unit sphere $\mathbb{S}^{2}$.

Proof. Using the same discussion as in Theorem 4.8, we deduce

$$
\sup _{M_{t}}|X| \leq \inf _{M_{t}}|X|+c .
$$

Thus, as $\sup _{M_{t}}|X| \rightarrow+\infty$ for $t \rightarrow+\infty$, we obtain that $\inf _{M_{t}}|X| \rightarrow+\infty$ for $t \rightarrow+\infty$.

For the sake of finishing our proof, we need a lemma as follows.

Sublemma. Under the assumptions of Theorem 6.1, assuming the origin lies inside $M_{0}$, there exists a positive constant $\rho$ such that the spheres $\partial B_{\rho(t)}(0)$ which solve (1.1) with initial radius $\rho$ intersect the surfaces $M_{t}$ which solve (1.1) with initial surface $M_{0}$ during the flow.

Proof of the Sublemma. Choose $\rho_{n}^{+}$to be the minimal radius of a sphere, centered at the origin, that encloses $M_{t}$ when $t=n$. Similarly, choose $\rho_{n}^{-}$to be the maximal radius of a sphere, centered at the origin, that is enclosed by $M_{t}$ when $t=n$, where $n=0,1,2,3, \cdots$. Correspondingly, we introduce $\partial B_{r_{n}^{+}(t)}$ as the sphere solving (1.1) and satisfying $r_{n}^{+}(n)=\rho_{n}^{+}$. We also introduce $\partial B_{r_{n}^{-}(t)}$ as the sphere solving (1.1) and satisfying $r_{n}^{-}(n)=\rho_{n}^{-}$. According to the containment principle, we deduce that $\left\{r_{n}^{+}(0)\right\}_{n=0}^{+\infty}$ is a non-increasing sequence. Hence a limit exists, say $r_{n}^{+}(0) \rightarrow r^{+}$. For the same reason, we obtain that $\left\{r_{n}^{-}(0)\right\}_{n=0}^{+\infty}$ is a non-decreasing sequence. Hence a limit exists, say $r_{n}^{-}(0) \rightarrow r^{-}$. By simple discussion, one can get $r^{-} \leq r^{+}$. Choose $\rho \in\left[r^{-}, r^{+}\right]$. We assert $\rho$ is the positive constant we want to find, and $\partial B_{\rho(t)}(0)$ which solve (1.1) with initial radius $\rho$ intersect the surfaces $M_{t}$ during our flow. Hence, we finish our proof of this sublemma.

Now let's continue our proof of Theorem 6.1. Combining the Sublemma above with equation (6.3),

$$
\sup _{M_{t}}|X|-c \leq\left\{(1-2 \alpha) t+\rho^{1-2 \alpha}\right\}^{\frac{1}{1-2 \alpha}} \leq \inf _{M_{t}}|X|+c, \text { when } 0<\alpha \leq \frac{1}{2},
$$

and

follow.

$$
\sup _{M_{t}}|X|-c \leq \rho e^{t} \leq \inf _{M_{t}}|X|+c, \text { when } \alpha=\frac{1}{2}
$$

Relations (6.1) and (6.2) come to light as consequences immediately. 
The Sublemma just tells us that such a $\rho$ exists. In fact, it is unique and, furthermore, the two limits we obtain in the Sublemma are equal, i.e. $r^{-}=r^{+}$. We derive this conclusion by

$$
\begin{gathered}
\left|\rho_{1} e^{t}-\rho_{2} e^{t}\right| \rightarrow+\infty, \\
\mid\left\{(1-2 \alpha) t+\rho_{1}^{1-2 \alpha}\right\}^{\frac{1}{1-2 \alpha}}-\left\{(1-2 \alpha) t+\rho_{2}^{1-2 \alpha}\right\}^{\frac{1}{1-2 \alpha} \mid \rightarrow+\infty,}
\end{gathered}
$$

if $\rho_{1} \neq \rho_{2}$, and our oscillation estimate

$$
\sup _{M_{t}}|X| \leq \inf _{M_{t}}|X|+c
$$

Our theorem follows.

Thus we have obtained our result.

Theorem 6.3. For any smooth closed strictly convex surface $M$ in $\mathbb{R}^{3}$, there exists a smooth family of surfaces $M_{t}, t \in[0,+\infty)$, solving equation (1.1) with $M_{0}=M$ when $0<\alpha \leq \frac{1}{2}$. For $t \rightarrow+\infty$, the surfaces $M_{t}$ converge to infinity. Furthermore, we obtain the following:

(i) If $0<\alpha<\frac{1}{2}$, there exists a unique positive constant $\rho$ such that the rescaled surfaces $\tilde{M}_{t}=\left\{(1-2 \alpha) t+\rho^{1-2 \alpha}\right\}^{-\frac{1}{1-2 \alpha}} M_{t}$ converge to the unit sphere $\mathbb{S}^{2}$.

(ii) If $\alpha=\frac{1}{2}$, there exists a unique positive constant $\rho$ such that the rescaled surfaces $\tilde{M}_{t}=\rho^{-1} e^{-t} M_{t}$ converge to the unit sphere $\mathbb{S}^{2}$.

Proof. This is a direct consequence. The smooth convergence follows by the same reason as in the last section. We omit the proof.

\section{ACKNOWLEDGEMENTS}

The author would like to thank the referee for the careful review and for providing a number of informative comments. The referee suggested an alternative and much more accessible analysis in Section 5, which has already been adopted in this paper. The author is glad to show an intense appreciation to Professor Weimin Sheng for his encouragement and instructive discussion. The author is also glad to thank Chao $\mathrm{Wu}$ for his help.

\section{REFERENCES}

[1] B. Andrews, Gauss curvature flow: the fate of the rolling stones, Invent. Math. 138(1999), no. 1, 151-161. MR.1714339 (2000i:53097)

[2] B. Andrews, Contraction of convex hypersurfaces in Euclidean space, Calc. Var. Partial Differential Equations 2(1994), no. 2, 151-171. MR1385524 (97b:53012)

[3] B. Chow, Deforming convex hypersurfaces by the n-th root of the Gaussian curvature, J. Differential Geom. 22(1985) 117-138. MR826427 (87f:58155)

[4] B. Chow and Peng Lu, The maximum principle for systems of parabolic equations subject to an avoidance set, Pacific J. Math. 214(2004), no. 2, 201-222. MR2042930 (2004m:35027)

[5] B. Chow and Robert Gulliver, Aleksandrov reflection and nonlinear evolution equations. I. The n-sphere and n-ball, Calc. Var. Partial Differential Equations 4(1996), no. 3, 249-264. MR:1386736 (97f:53064)

[6] W. Firey, Shapes of worn stones, Mathmatika 21(1974), 1-11. MR0362045 (50:14487)

[7] C. Gerhardt, Closed Weingarten hypersurfaces in Riemannian manifolds, J. Differential Geom. 43(1996), no. 3, 612-641. MR.1412678 (97g:53067)

[8] R. S. Hamilton, Three-manifolds with positive Ricci curvature, J. Differential Geom. 17(1982), no. 2, 255-306. MR664497 (84a:53050)

[9] G. Huisken, Flow by mean curvature of convex surfaces into spheres, J. Differential Geom. 20(1984), no. 1, 237-266. MR772132 (86j:53097) 
[10] G. Huisken and Alexander Polden, Geometric evolution equations for hypersurfaces, Calculus of variations and geometric problems (Cetraro, 1996), Lecture Notes in Math., vol. 1713, Springer, Berlin, 1999, pp. 45-84. MR.1731639(2000j:53090)

[11] G. Huisken, On the expansion of convex hypersurfaces by the inverse of symmetric curvature functions (unpublished).

[12] J. A. McCoy, The surface area preserving mean curvature flow, Asian J. Math. 7(2003), no. 1, 7-30. MR2015239 (2004m:53117)

[13] Oliver C. Schnürer, Surfaces expanding by the inverse Gauss curvature flow, J. Reine Angew. Math. 600(2006), 117-134. MR2283800 (2007j:53074)

[14] Kaising Tso, Deforming a hypersurface by its Gauss-Kronecker curvature, Comm. Pure Appl. Math. 38(1985), no. 6, 867-882. MR812353 (87e:53009)

[15] J. Urbas, Complete noncompact self-similar solutions of Gauss curvature flows II. Negative powers, Advances in Differential Equations 4(1999), no. 3, 323-346. MR.1671253 (2000a:53117)

[16] J. Urbas, An expansion of convex hypersurfaces, J. Differential Geom. 33(1991), no. 1, 91-125. MR.1085136(91j:58155)

Department of Mathematics, Zhejiang University, Hangzhou 310027, People's RepubLIC OF CHINA

E-mail address: 85lqr@163.com 